\title{
GENERAL THREE POINTS INEQUALITIES FOR WEIGHTED RIEMANN-STIELTJES INTEGRAL
}

\author{
SILVESTRU SEVER DRAGOMIR ${ }^{1,2}$
}

\begin{abstract}
In this paper we provide amongst others some simple error bounds in approximating the weighted Riemann-Stieltjes integral $\int_{a}^{b} f(t) g(t) d v(t)$ by the use of three points formula

$$
f(b) \int_{c}^{b} g(s) d v(s)+f(a) \int_{a}^{d} g(s) d v(s)-f(x) \int_{c}^{d} g(t) d v(t)
$$

where $x, c, d \in[a, b], g, v:[a, b] \rightarrow \mathbb{C}$ under bounded variation and Lipschitzian assumptions for the function $f$ and such that the involved Riemann-Stieltjes integrals exist.
\end{abstract}

\section{Introduction}

Assume that $u, f:[a, b] \rightarrow \mathbb{C}$ are bounded. If the Riemann-Stieltjes integral $\int_{a}^{b} f(t) d u(t)$ exists, we write for simplicity, like in [1, p. 142] that $f \in \mathcal{R}_{\mathbb{C}}(u,[a, b])$, or $\mathcal{R}_{\mathbb{C}}(u)$ when the interval is implicitly known. If the functions $u, f$ are real valued, then we write $f \in \mathcal{R}(u,[a, b])$, or $\mathcal{R}(u)$.

In order to approximate the Riemann-Stieltjes integral $\int_{a}^{b} f(t) d u(t)$ by the use of a three points formula, namely to establish bounds for the error functional

$$
\begin{aligned}
T \Theta(f, u ; a, b, x, \alpha):=(1-\alpha)\{[u(b) & -u(x)] f(b)+[u(x)-u(a)] f(a)\} \\
& +\alpha[u(b)-u(a)] f(x)-\int_{a}^{b} f(t) d u(t),
\end{aligned}
$$

where $\alpha \in[0,1]$ and $x \in[a, b]$, under bounded variation assumptions for the functions $u$ and $f$ and such that the involved Riemann-Stieltjes integral exists, in the recent paper [26] we have obtained the following result:

Theorem 1. Let $f, u:[a, b] \rightarrow \mathbb{C}$ and $x \in[a, b]$ are such that $f \in \mathcal{R}_{\mathbb{C}}(u,[a, b])$. If $f$ and $u$ are of bounded variation, then

$$
\begin{aligned}
& |T \Theta(f, u ; a, b, x, \alpha)| \\
& \leq \alpha\left[\int_{a}^{x}\left(\bigvee_{a}^{t}(u)\right) d\left(\bigvee_{a}^{t}(f)\right)+\int_{x}^{b}\left(\bigvee_{t}^{b}(u)\right) d\left(\bigvee_{a}^{t}(f)\right)\right] \\
& \quad+(1-\alpha)\left[\int_{a}^{x}\left(\bigvee_{t}^{x}(u)\right) d\left(\bigvee_{a}^{t}(f)\right)+\int_{x}^{b}\left(\bigvee_{x}^{t}(u)\right) d\left(\bigvee_{a}^{t}(f)\right)\right]
\end{aligned}
$$

1991 Mathematics Subject Classification. 26D15, 26D10, 47A63, 47A30.

Key words and phrases. Riemann-Stieltjes integral, Continuous functions, Functions of bounded variation, Lipschitzian functions. 


$$
\begin{aligned}
\leq \max \{\alpha, 1-\alpha\} & \left(\bigvee_{a}^{x}(u) \bigvee_{a}^{x}(f)+\bigvee_{x}^{b}(u) \bigvee_{x}^{b}(f)\right) \\
\leq \frac{1}{2} \max \{\alpha, 1-\alpha\} & \left\{\begin{array}{l}
\left(\bigvee_{a}^{b}(u)+\left|\bigvee_{a}^{x}(u)-\bigvee_{x}^{b}(u)\right|\right) \bigvee_{a}^{b}(f) \\
\left(\bigvee_{a}^{b}(f)+\left|\bigvee_{a}^{x}(f)-\bigvee_{x}^{b}(f)\right|\right) \bigvee_{a}^{b}(u) \\
\leq \max \{\alpha, 1-\alpha\} \bigvee_{a}^{b}(u) \bigvee_{a}^{b}(f) .
\end{array}\right.
\end{aligned}
$$

In [27] we also obtained the following result in the case of Lipschitzian integrands:

Theorem 2. Let $f, u:[a, b] \rightarrow \mathbb{C}$ and $x \in[a, b]$. If $f$ is Lipschitzian with the constant $L>0$, namely

$$
|f(t)-f(s)| \leq L|t-s| \text { for all } t, s \in[a, b]
$$

and $u$ is of bounded variation, then $f \in \mathcal{R}_{\mathbb{C}}(u,[a, b])$ and

$$
\begin{array}{r}
|T \Theta(f, u ; a, b, x, \alpha)| \\
\leq \alpha L\left[\int_{a}^{x}\left(\bigvee_{a}^{t}(u)\right) d t+\int_{x}^{b}\left(\bigvee_{t}^{b}(u)\right) d t\right] \\
+(1-\alpha) L\left[\int_{a}^{x}\left(\bigvee_{t}^{x}(u)\right) d t+\int_{x}^{b}\left(\bigvee_{x}^{t}(u)\right) d t\right] \\
\leq \max \{\alpha, 1-\alpha\} L\left((x-a) \bigvee_{a}^{x}(u)+(b-x) \bigvee_{x}^{b}(u)\right) \\
\leq \frac{1}{2} \max \{\alpha, 1-\alpha\} L\left\{\begin{array}{c}
\left(\bigvee_{a}^{b}(u)+\left|\bigvee_{a}^{x}(u)-\bigvee_{x}^{b}(u)\right|\right)(b-a) \\
(b-a+|2 x-a-b|) \bigvee_{a}^{b}(u)
\end{array}\right. \\
\leq \max \{\alpha, 1-\alpha\} L(b-a) \bigvee_{a}^{b}(u) .
\end{array}
$$

For various bounds on the error functional

$$
D(f, u ; a, b):=\int_{a}^{b} f(t) d u(t)-\frac{1}{b-a}[u(b)-u(a)] \cdot \int_{a}^{b} f(t) d t
$$

where $f$ and $u$ belong to different classes of function for which the Riemann-Stieltjes integral exists, see [22], [21], [20], and [8] and the references therein.

Bounds for the functional

$$
\Theta(f, u ; a, b, x):=\int_{a}^{b} f(t) d u(t)-f(x)[u(b)-u(a)]
$$


can be found in [15], [16] and [8], while for the functional

$$
T(f, u ; a, b, x):=\int_{a}^{b} f(t) d u(t)-[u(b)-u(x)] f(b)-[u(x)-u(a)] f(a)
$$

they may be found in [28], [8], [3] and [2]. The details are omitted.

In this paper we provide some simple error bounds in approximating the weighted Riemann-Stieltjes integral $\int_{a}^{b} f(t) g(t) d v(t)$ by the use of various general three points formulae out of which we mention the following one

$$
f(b) \int_{c}^{b} g(s) d v(s)+f(a) \int_{a}^{d} g(s) d v(s)-f(x) \int_{c}^{d} g(t) d v(t),
$$

where $x, c, d \in[a, b], g, v:[a, b] \rightarrow \mathbb{C}$ under bounded variation and Lipschitzian assumptions for the function $f$ and such that the involved Riemann-Stieltjes integral exist.

\section{Some Preliminary Facts}

The following properties of Riemann-Stieltjes integral are well know, [1, p. 158$159]$ :

Lemma 1. Assume that $f \in \mathcal{R}_{\mathbb{C}}(\alpha,[a, b])$ and $f \in \mathcal{R}_{\mathbb{C}}(\alpha,[a, b])$ where $\alpha \in$ $\mathcal{B} \mathcal{V}_{\mathbb{C}}[a, b]$, namely of bounded variation on $[a, b]$. Define

$$
F(x):=\int_{a}^{x} f(t) d \alpha(t)
$$

and

$$
G(x):=\int_{a}^{x} g(t) d \alpha(t)
$$

where $x \in[a, b]$.

Then $f \in \mathcal{R}_{\mathbb{C}}(G,[a, b]), g \in \mathcal{R}_{\mathbb{C}}(F,[a, b]), f g \in \mathcal{R}_{\mathbb{C}}(\alpha,[a, b])$ and we have

$$
\int_{a}^{b} f(t) g(t) d \alpha(t)=\int_{a}^{b} f(t) d G(t)=\int_{a}^{b} g(t) d F(t) .
$$

If $c \in[a, b]$ and consider the integral $\int_{c}^{t} g(s) d v(s)$ that is assumed to exist for any $t \in[a, b]$, then

$$
\int_{c}^{t} g(s) d v(s)=\int_{a}^{t} g(s) d v(s)-\int_{a}^{c} g(s) d v(s)
$$

for any $t \in[a, b]$.

Indeed if $t \in[c, b]$, then (2.1) is obvious. If $t \in[a, c]$, then

$$
\int_{a}^{c} g(s) d v(s)=\int_{a}^{t} g(s) d v(s)+\int_{t}^{c} g(s) d v(s),
$$

which also gives (2.1).

We start with the following simple fact: 
Preprints (www.preprints.org) | NOT PEER-REVIEWED | Posted: 4 September 2018

doi:10.20944/preprints201809.0060.v1

4

S. S. DRAGOMIR

Lemma 2. Let $f, g, v:[a, b] \rightarrow \mathbb{C}, \lambda, \mu \in \mathbb{C}$ and $x, c, d \in[a, b]$. If $g, f g \in$ $\mathcal{R}_{\mathbb{C}}(v,[a, b])$, then

$$
\begin{aligned}
\left(\int_{d}^{b} g(s) d v(s)-\mu\right) f(b)+\left(\lambda+\int_{a}^{c} g(t) d v(t)\right) f(a) \\
\quad+\left(\int_{c}^{d} g(t) d v(t)+\mu-\lambda\right) f(x)-\int_{a}^{b} f(t) g(t) d v(t) \\
\quad=\int_{a}^{x}\left(\int_{c}^{t} g(s) d v(s)-\lambda\right) d f(t)+\int_{x}^{b}\left(\int_{d}^{t} g(s) d v(s)-\mu\right) d f(t)
\end{aligned}
$$

In particular, for $\mu=\lambda$ we have

$$
\begin{gathered}
\left(\int_{d}^{b} g(s) d v(s)-\lambda\right) f(b)+\left(\lambda+\int_{a}^{c} g(t) d v(t)\right) f(a) \\
\quad+f(x) \int_{c}^{d} g(t) d v(t)-\int_{a}^{b} f(t) g(t) d v(t) \\
=\int_{a}^{x}\left(\int_{c}^{t} g(s) d v(s)-\lambda\right) d f(t)+\int_{x}^{b}\left(\int_{d}^{t} g(s) d v(s)-\lambda\right) d f(t) .
\end{gathered}
$$

Proof. Assume that $x, c, d \in[a, b]$. Using the integration by parts formula for the Riemann-Stieltjes integral and Lemma 1, we have

$$
\begin{gathered}
\text { (2.4) } \int_{a}^{x}\left(\int_{c}^{t} g(s) d v(s)-\lambda\right) d f(t) \\
=\left.\left(\int_{c}^{t} g(t) d v(t)-\lambda\right) f(t)\right|_{a} ^{x}-\int_{a}^{x} f(t) d\left(\int_{c}^{t} g(s) d v(s)-\lambda\right) \\
=\left(\int_{c}^{x} g(t) d v(t)-\lambda\right) f(x)-\left(\int_{c}^{a} g(t) d v(t)-\lambda\right) f(a)-\int_{a}^{x} f(t) g(t) d v(t) \\
=\left(\int_{c}^{x} g(t) d v(t)-\lambda\right) f(x)+\left(\lambda+\int_{a}^{c} g(t) d v(t)\right) f(a)-\int_{a}^{x} f(t) g(t) d v(t) .
\end{gathered}
$$

In a similar way,

$$
\begin{aligned}
& \text { (2.5) } \int_{x}^{b}\left(\int_{d}^{t} g(s) d v(s)-\mu\right) d f(t) \\
& =\left.\left(\int_{d}^{t} g(s) d v(s)-\mu\right) f(t)\right|_{x} ^{b}-\int_{x}^{b} f(t) d\left(\int_{d}^{t} g(s) d v(s)-\mu\right) \\
& =\left(\int_{d}^{b} g(s) d v(s)-\mu\right) f(b)-\left(\int_{d}^{x} g(s) d v(s)-\mu\right) f(x)-\int_{x}^{b} f(t) g(t) d v(t) \\
& =\left(\int_{d}^{b} g(s) d v(s)-\mu\right) f(b)+\left(\mu-\int_{d}^{x} g(s) d v(s)\right) f(x)-\int_{x}^{b} f(t) g(t) d v(t) .
\end{aligned}
$$


If we add (2.4) and (2.5), we get

$$
\begin{gathered}
\int_{a}^{x}\left(\int_{c}^{t} g(s) d v(s)-\lambda\right) d f(t)+\int_{x}^{b}\left(\int_{d}^{t} g(s) d v(s)-\mu\right) d f(t) \\
=\left(\int_{c}^{x} g(t) d v(t)-\lambda\right) f(x)+\left(\lambda+\int_{a}^{c} g(t) d v(t)\right) f(a) \\
+\left(\int_{d}^{b} g(s) d v(s)-\mu\right) f(b)+\left(\mu-\int_{d}^{x} g(s) d v(s)\right) f(x)-\int_{a}^{b} f(t) g(t) d v(t),
\end{gathered}
$$

which is equivalent to the desired result $(2.2)$

If we take $d=c$ above, we get:

Corollary 1. Let $f, g, v, f:[a, b] \rightarrow \mathbb{C}, \lambda, \mu \in \mathbb{C}$ and $x, c \in[a, b]$. If $g, f g \in$ $\mathcal{R}_{\mathbb{C}}(v,[a, b])$, then

$$
\begin{gathered}
\left(\int_{c}^{b} g(s) d v(s)-\mu\right) f(b)+\left(\lambda+\int_{a}^{c} g(t) d v(t)\right) f(a) \\
+(\mu-\lambda) f(x)-\int_{a}^{b} f(t) g(t) d v(t) \\
=\int_{a}^{x}\left(\int_{c}^{t} g(s) d v(s)-\lambda\right) d f(t)+\int_{x}^{b}\left(\int_{c}^{t} g(s) d v(s)-\mu\right) d f(t) .
\end{gathered}
$$

In particular, for $\mu=\lambda$ we have

$$
\begin{aligned}
\left(\int_{c}^{b} g(s) d v(s)-\lambda\right) f(b)+\left(\lambda+\int_{a}^{c} g(t) d v(t)\right) f(a) \\
-\int_{a}^{b} f(t) g(t) d v(t)=\int_{a}^{b}\left(\int_{c}^{t} g(s) d v(s)-\lambda\right) d f(t) .
\end{aligned}
$$

Remark 1. If we take $g(t)=1, t \in[a, b]$ in (2.2) and (2.3), then we get

$$
\begin{aligned}
& (v(b)-v(d)-\mu) f(b)+(\lambda+v(c)-v(a)) f(a) \\
& +(v(d)+\mu-\lambda-v(c)) f(x)-\int_{a}^{b} f(t) d v(t) \\
& \quad=\int_{a}^{x}(v(t)-v(c)-\lambda) d f(t)+\int_{x}^{b}(v(t)-v(d)-\mu) d f(t) .
\end{aligned}
$$

If we take $\beta=v(d)+\mu$ and $\gamma=\lambda+v(c)$, then by (2.8) we get

$$
\begin{aligned}
(v(b)-\beta) f(b)+(\gamma-v(a)) & f(a)+(\beta-\gamma) f(x)-\int_{a}^{b} f(t) d v(t) \\
= & \int_{a}^{x}(v(t)-\gamma) d f(t)+\int_{x}^{b}(v(t)-\beta) d f(t) .
\end{aligned}
$$

In particular, for $\beta=\gamma$ we get by (2.9) that

$$
(v(b)-\beta) f(b)+(\beta-v(a)) f(a)-\int_{a}^{b} f(t) d v(t)=\int_{a}^{b}(v(t)-\beta) d f(t) .
$$


Remark 2. If we take $c=b$ and $d=a$ in Lemma 2, then we get

$$
\begin{aligned}
& \left(\int_{a}^{b} g(s) d v(s)-\mu\right) f(b)+\left(\lambda+\int_{a}^{b} g(t) d v(t)\right) f(a) \\
& \quad+\left(\mu-\lambda-\int_{a}^{b} g(t) d v(t)\right) f(x)-\int_{a}^{b} f(t) g(t) d v(t) \\
& \quad=\int_{x}^{b}\left(\int_{a}^{t} g(s) d v(s)-\mu\right) d f(t)-\int_{a}^{x}\left(\int_{t}^{b} g(s) d v(s)+\lambda\right) d f(t) .
\end{aligned}
$$

In particular, for $\mu=\lambda$ we obtain

$$
\begin{gathered}
\left(\int_{a}^{b} g(s) d v(s)-\lambda\right) f(b)+\left(\lambda+\int_{a}^{b} g(t) d v(t)\right) f(a) \\
-f(x) \int_{a}^{b} g(t) d v(t)-\int_{a}^{b} f(t) g(t) d v(t) \\
=\int_{x}^{b}\left(\int_{a}^{t} g(s) d v(s)-\lambda\right) d f(t)-\int_{a}^{x}\left(\int_{t}^{b} g(s) d v(s)+\lambda\right) d f(t) .
\end{gathered}
$$

If we take $c=a$ and $d=b$ in Lemma 2, then we get

$$
\begin{aligned}
& \lambda f(a)-\mu f(b)+(\mu-\lambda) f(x)-\int_{a}^{b} f(t) g(t) d v(t) \\
& \quad=\int_{a}^{x}\left(\int_{a}^{t} g(s) d v(s)-\lambda\right) d f(t)-\int_{x}^{b}\left(\int_{t}^{b} g(s) d v(s)+\mu\right) d f(t) .
\end{aligned}
$$

In particular, for $\mu=\lambda$ we obtain

$$
\begin{aligned}
& \lambda f(a)-\lambda f(b)-\int_{a}^{b} f(t) g(t) d v(t) \\
& \quad=\int_{a}^{x}\left(\int_{a}^{t} g(s) d v(s)-\lambda\right) d f(t)-\int_{x}^{b}\left(\int_{t}^{b} g(s) d v(s)+\lambda\right) d f(t) .
\end{aligned}
$$

Remark 3. If we take $g(t)=1, t \in[a, b]$ in (2.11), then we get

$$
\begin{aligned}
& (v(b)-v(a)-\mu) f(b)+(\lambda+v(b)-v(a)) f(a) \\
& +(\mu-\lambda-v(b)+v(a)) f(x)-\int_{a}^{b} f(t) d v(t) \\
& \quad=\int_{x}^{b}(v(t)-v(a)-\mu) d f(t)-\int_{a}^{x}(v(b)-v(t)+\lambda) d f(t) .
\end{aligned}
$$

If this equality we take $\mu=\lambda$, then we get

$$
\begin{aligned}
(v(b)-v(a)- & \lambda) f(b)+(\lambda+v(b)-v(a)) f(a) \\
& -(v(b)-v(a)) f(x)-\int_{a}^{b} f(t) d v(t) \\
= & \int_{x}^{b}(v(t)-v(a)-\lambda) d f(t)-\int_{a}^{x}(v(b)-v(t)+\lambda) d f(t) .
\end{aligned}
$$


Remark 4. If we take $\mu=-\int_{a}^{d} g(s) d v(s)$ and $\lambda=\int_{c}^{b} g(t) d v(t)$ in (2.2), then we get

$$
\begin{gathered}
\int_{a}^{b} g(s) d v(s)(f(b)+f(a)) \\
+\left(\int_{c}^{x} g(t) d v(t)-\int_{d}^{x} g(s) d v(s)-\int_{a}^{d} g(s) d v(s)-\int_{c}^{b} g(t) d v(t)\right) f(x) \\
-\int_{a}^{b} f(t) g(t) d v(t) \\
=\int_{a}^{x}\left(\int_{c}^{t} g(s) d v(s)-\int_{c}^{b} g(t) d v(t)\right) d f(t) \\
\quad+\int_{x}^{b}\left(\int_{d}^{t} g(s) d v(s)+\int_{a}^{d} g(s) d v(s)\right) d f(t)
\end{gathered}
$$

which is equivalent to

$$
\begin{aligned}
{[f(b)+f(a)} & -f(x)] \int_{a}^{b} g(s) d v(s)-\int_{a}^{b} f(t) g(t) d v(t) \\
& =\int_{x}^{b}\left(\int_{a}^{t} g(s) d v(s)\right) d f(t)-\int_{a}^{x}\left(\int_{t}^{b} g(s) d v(s)\right) d f(t) .
\end{aligned}
$$

If we take $\lambda=\int_{c}^{d} g(t) d v(t)$ and $\mu=-\int_{c}^{d} g(t) d v(t)$, then by (2.2) we get

$$
\begin{gathered}
\left(\int_{d}^{b} g(s) d v(s)+\int_{c}^{d} g(t) d v(t)\right) f(b) \\
+\left(\int_{c}^{d} g(t) d v(t)+\int_{a}^{c} g(t) d v(t)\right) f(a) \\
+\left(\int_{c}^{x} g(t) d v(t)-\int_{d}^{x} g(s) d v(s)-\int_{c}^{d} g(t) d v(t)-\int_{c}^{d} g(t) d v(t)\right) f(x) \\
=\int_{a}^{x}\left(\int_{c}^{t} g(s) d v(s)-\int_{c}^{b} g(t) d v(t)\right) d f(t) \\
+\int_{x}^{b}\left(\int_{d}^{t} g(s) d v(s)+\int_{c}^{d} g(t) d v(t)\right) d f(t)
\end{gathered}
$$


which is equivalent to

$$
\begin{gathered}
f(b) \int_{c}^{b} g(s) d v(s)+f(a) \int_{a}^{d} g(t) d v(t)-f(x) \int_{c}^{d} g(t) d v(t) \\
-\int_{a}^{b} f(t) g(t) d v(t) \\
=\int_{a}^{x}\left(\int_{d}^{t} g(s) d v(s)\right) d f(t)+\int_{x}^{b}\left(\int_{c}^{t} g(s) d v(s)\right) d f(t) .
\end{gathered}
$$

Remark 5. If we take $\mu=\int_{d}^{b} g(s) d v(s)$ and $\lambda=-\int_{a}^{c} g(t) d v(t)$ in (2.2), then we get

$$
\begin{gathered}
\left(\int_{c}^{x} g(t) d v(t)+\int_{x}^{d} g(s) d v(s)+\int_{d}^{b} g(s) d v(s)+\int_{a}^{c} g(t) d v(t)\right) f(x) \\
-\int_{a}^{b} f(t) g(t) d v(t) \\
=\int_{a}^{x}\left(\int_{c}^{t} g(s) d v(s)+\int_{a}^{c} g(t) d v(t)\right) d f(t) \\
+\int_{x}^{b}\left(\int_{d}^{t} g(s) d v(s)-\int_{d}^{b} g(s) d v(s)\right) d f(t),
\end{gathered}
$$

which is equivalent to

$$
\begin{aligned}
& f(x) \int_{a}^{b} g(t) d v(t)-\int_{a}^{b} f(t) g(t) d v(t) \\
&=\int_{a}^{x}\left(\int_{a}^{t} g(s) d v(s)\right) d f(t)-\int_{x}^{b}\left(\int_{t}^{b} g(s) d v(s)\right) d f(t) .
\end{aligned}
$$

\section{Inequalities for Integrands of Bounded Variation}

We have:

Theorem 3. Assume that $f:[a, b] \rightarrow \mathbb{C}$ is of bounded variation, $g, v:[a, b] \rightarrow \mathbb{C}$, $\lambda, \mu \in \mathbb{C}$ and $x, c, d \in[a, b]$ are such that the Riemann-Stieltjes integrals below exist. Then

$$
\begin{aligned}
& \mid\left(\int_{d}^{b} g(s) d v(s)-\mu\right) f(b)+\left(\lambda+\int_{a}^{c} g(t) d v(t)\right) f(a) \\
& +\left(\int_{c}^{d} g(t) d v(t)+\mu-\lambda\right) f(x)-\int_{a}^{b} f(t) g(t) d v(t) \mid \\
& \leq \max _{t \in[a, x]}\left|\int_{c}^{t} g(s) d v(s)-\lambda\right| \bigvee_{a}^{x}(f)+\max _{t \in[x, b]}\left|\int_{d}^{t} g(s) d v(s)-\mu\right| \bigvee_{x}^{b}(f) \\
& \quad \leq \max \left\{\max _{t \in[a, x]}\left|\int_{c}^{t} g(s) d v(s)-\lambda\right|, \max _{t \in[x, b]}\left|\int_{d}^{t} g(s) d v(s)-\mu\right|\right\} \bigvee_{a}^{b}(f) .
\end{aligned}
$$


In particular, for $\mu=\lambda$ we have

$$
\begin{aligned}
& \mid\left(\int_{d}^{b} g(s) d v(s)-\lambda\right) f(b)+\left(\lambda+\int_{a}^{c} g(t) d v(t)\right) f(a) \\
& \quad+f(x) \int_{c}^{d} g(t) d v(t)-\int_{a}^{b} f(t) g(t) d v(t) \mid \\
& \leq \max _{t \in[a, x]}\left|\int_{c}^{t} g(s) d v(s)-\lambda\right| \bigvee_{a}^{x}(f)+\max _{t \in[x, b]}\left|\int_{d}^{t} g(s) d v(s)-\lambda\right| \bigvee_{x}^{b}(f) \\
& \leq \max _{t}\left\{\max _{t \in[a, x]}\left|\int_{c}^{t} g(s) d v(s)-\lambda\right|, \max _{t \in[x, b]}\left|\int_{d}^{t} g(s) d v(s)-\lambda\right|\right\} \bigvee_{a}^{b}(f) .
\end{aligned}
$$

Proof. It is well known that, if $p:[a, b] \rightarrow \mathbb{C}$ is continuous and $v:[a, b] \rightarrow \mathbb{C}$ of bounded variation, then [1]

$$
\left|\int_{a}^{b} p(t) d v(t)\right| \leq \int_{a}^{b}|p(t)| d\left(\bigvee_{a}^{t}(v)\right) \leq \max _{t \in[a, b]}|p(t)| \bigvee_{a}^{b}(v)
$$

By using the identity (2.2) and the property (3.3) we get

$$
\begin{aligned}
& \mid\left(\int_{d}^{b} g(s) d v(s)-\mu\right) f(b)+\left(\lambda+\int_{a}^{c} g(t) d v(t)\right) f(a) \\
& \quad+\left(\int_{c}^{d} g(t) d v(t)+\mu-\lambda\right) f(x)-\int_{a}^{b} f(t) g(t) d v(t) \mid \\
& \leq\left|\int_{a}^{x}\left(\int_{c}^{t} g(s) d v(s)-\lambda\right) d f(t)\right|+\left|\int_{x}^{b}\left(\int_{d}^{t} g(s) d v(s)-\mu\right) d f(t)\right| \\
& \leq \int_{a}^{x}\left|\int_{c}^{t} g(s) d v(s)-\lambda\right| d\left(\bigvee_{a}^{t}(f)\right)+\int_{x}^{b}\left|\int_{d}^{t} g(s) d v(s)-\mu\right| d\left(\bigvee_{x}^{t}(f)\right) \\
& \leq \max _{t \in[a, x]}\left|\int_{c}^{t} g(s) d v(s)-\lambda\right| \bigvee_{a}^{x}(f)+\max _{t \in[x, b]}\left|\int_{d}^{t} g(s) d v(s)-\mu\right| \bigvee_{x}^{b}(f),
\end{aligned}
$$

which proves the first inequality in (3.1).

Observe that

$$
\begin{array}{r}
\max _{t \in[a, x]}\left|\int_{c}^{t} g(s) d v(s)-\lambda\right| \bigvee_{a}^{x}(f)+\max _{t \in[x, b]}\left|\int_{d}^{t} g(s) d v(s)-\mu\right| \bigvee_{x}^{b}(f) \\
\leq \max \left\{\max _{t \in[a, x]}\left|\int_{c}^{t} g(s) d v(s)-\lambda\right|, \max _{t \in[x, b]}\left|\int_{d}^{t} g(s) d v(s)-\mu\right|\right\}\left(\bigvee_{a}^{x}(f)+\bigvee_{x}^{b}(f)\right) \\
=\max \left\{\max _{t \in[a, x]}\left|\int_{c}^{t} g(s) d v(s)-\lambda\right|, \max _{t \in[x, b]}\left|\int_{d}^{t} g(s) d v(s)-\mu\right|\right\} \bigvee_{a}^{b}(f),
\end{array}
$$

which proves the last part of (3.1). 
Preprints (www.preprints.org) | NOT PEER-REVIEWED | Posted: 4 September 2018

doi:10.20944/preprints201809.0060.v1

10

S. S. DRAGOMIR

Remark 6. If $m \in(a, b)$ is such that $\bigvee_{a}^{m}(f)=\bigvee_{m}^{b}(f)$, then under the assumptions of Theorem 3 we have the inequalities

$$
\begin{aligned}
& \mid\left(\int_{d}^{b} g(s) d v(s)-\mu\right) f(b)+\left(\lambda+\int_{a}^{c} g(t) d v(t)\right) f(a) \\
& \quad+\left(\int_{c}^{d} g(t) d v(t)+\mu-\lambda\right) f(m)-\int_{a}^{b} f(t) g(t) d v(t) \mid \\
& \quad \leq \frac{1}{2}\left[\max _{t \in[a, m]}\left|\int_{c}^{t} g(s) d v(s)-\lambda\right|+\max _{t \in[m, b]}\left|\int_{d}^{t} g(s) d v(s)-\mu\right| \mid \bigvee_{a}^{b}(f)\right.
\end{aligned}
$$

and

$$
\begin{aligned}
& \mid\left(\int_{d}^{b} g(s) d v(s)-\lambda\right) f(b)+\left(\lambda+\int_{a}^{c} g(t) d v(t)\right) f(a) \\
& \quad+f(m) \int_{c}^{d} g(t) d v(t)-\int_{a}^{b} f(t) g(t) d v(t) \mid \\
& \quad \leq \frac{1}{2}\left[\max _{t \in[a, m]}\left|\int_{c}^{t} g(s) d v(s)-\lambda\right|+\max _{t \in[m, b]}\left|\int_{d}^{t} g(s) d v(s)-\lambda\right|\right] \bigvee_{a}^{b}(f) .
\end{aligned}
$$

Corollary 2. With the assumptions of Theorem 3, and if $d=c$, then

$$
\begin{aligned}
& \mid\left(\int_{c}^{b} g(s) d v(s)-\mu\right) f(b)+\left(\lambda+\int_{a}^{c} g(t) d v(t)\right) f(a) \\
& +(\mu-\lambda) f(x)-\int_{a}^{b} f(t) g(t) d v(t) \mid \\
& \leq \max _{t \in[a, x]}\left|\int_{c}^{t} g(s) d v(s)-\lambda\right| \bigvee_{a}^{x}(f)+\max _{t \in[x, b]}\left|\int_{c}^{t} g(s) d v(s)-\mu\right| \bigvee_{x}^{b}(f) \\
& \leq \max \left\{\max _{t \in[a, x]}\left|\int_{c}^{t} g(s) d v(s)-\lambda\right|, \max _{t \in[x, b]}\left|\int_{c}^{t} g(s) d v(s)-\mu\right|\right\} \bigvee_{a}^{b}(f) .
\end{aligned}
$$

In particular, for $\mu=\lambda$, we get

$$
\begin{aligned}
& \mid\left(\int_{c}^{b} g(s) d v(s)-\lambda\right) f(b)+\left(\lambda+\int_{a}^{c} g(t) d v(t)\right) f(a) \\
& -\int_{a}^{b} f(t) g(t) d v(t) \mid \\
& \leq \max _{t \in[a, x]}\left|\int_{c}^{t} g(s) d v(s)-\lambda\right| \bigvee_{a}^{x}(f)+\max _{t \in[x, b]}\left|\int_{c}^{t} g(s) d v(s)-\lambda\right| \bigvee_{x}^{b}(f) \\
& \quad \leq \max \left\{\max _{t \in[a, x]}\left|\int_{c}^{t} g(s) d v(s)-\lambda\right|, \max _{t \in[x, b]}\left|\int_{c}^{t} g(s) d v(s)-\lambda\right|\right\} \bigvee_{a}^{b}(f) .
\end{aligned}
$$


Remark 7. If we take $c=x$ in Corollary 2, then we get

$$
\begin{aligned}
& \mid\left(\int_{x}^{b} g(s) d v(s)-\mu\right) f(b)+\left(\lambda+\int_{a}^{x} g(t) d v(t)\right) f(a) \\
& +(\mu-\lambda) f(x)-\int_{a}^{b} f(t) g(t) d v(t) \mid \\
& \leq \max _{t \in[a, x]}\left|\int_{x}^{t} g(s) d v(s)-\lambda\right| \bigvee_{a}^{x}(f)+\max _{t \in[x, b]}\left|\int_{x}^{t} g(s) d v(s)-\mu\right| \bigvee_{x}^{b}(f) \\
& \leq \max \left\{\max _{t \in[a, x]}\left|\int_{x}^{t} g(s) d v(s)-\lambda\right|, \max _{t \in[x, b]}\left|\int_{x}^{t} g(s) d v(s)-\mu\right|\right\} \bigvee_{a}^{b}(f) .
\end{aligned}
$$

In particular, for $\mu=\lambda$, we get

$$
\begin{aligned}
& \mid\left(\int_{x}^{b} g(s) d v(s)-\lambda\right) f(b)+\left(\lambda+\int_{a}^{x} g(t) d v(t)\right) f(a) \\
& -\int_{a}^{b} f(t) g(t) d v(t) \mid \\
& \leq \max _{t \in[a, x]}\left|\int_{x}^{t} g(s) d v(s)-\lambda\right| \bigvee_{a}^{x}(f)+\max _{t \in[x, b]}\left|\int_{x}^{t} g(s) d v(s)-\lambda\right| \bigvee_{x}^{b}(f) \\
& \leq \max _{t \in[a, b]}\left|\int_{x}^{t} g(s) d v(s)-\lambda\right| \bigvee_{a}^{b}(f) .
\end{aligned}
$$

Corollary 3. With the assumptions of Theorem 3, and if $c=b$ and $d=a$, then

$$
\begin{aligned}
& \mid\left(\int_{a}^{b} g(s) d v(s)-\mu\right) f(b)+\left(\lambda+\int_{a}^{b} g(t) d v(t)\right) f(a) \\
& +\left(\mu-\lambda-\int_{a}^{b} g(t) d v(t)\right) f(x)-\int_{a}^{b} f(t) g(t) d v(t) \mid \\
& \leq \max _{t \in[a, x]}\left|\int_{t}^{b} g(s) d v(s)+\lambda\right| \bigvee_{a}^{x}(f)+\max _{t \in[x, b]}\left|\int_{a}^{t} g(s) d v(s)-\mu\right| \bigvee_{x}^{b}(f) \\
& \leq \max \left\{\max _{t \in[a, x]}\left|\int_{t}^{b} g(s) d v(s)+\lambda\right|, \max _{t \in[x, b]}\left|\int_{a}^{t} g(s) d v(s)-\mu\right|\right\} \bigvee_{a}^{b}(f) .
\end{aligned}
$$


In particular, for $\mu=\lambda$ we have

$$
\begin{aligned}
& \quad \mid\left(\int_{a}^{b} g(s) d v(s)-\lambda\right) f(b)+\left(\lambda+\int_{a}^{b} g(t) d v(t)\right) f(a) \\
& \quad-\int_{a}^{b} g(t) d v(t) f(x)-\int_{a}^{b} f(t) g(t) d v(t) \mid \\
& \leq \max _{t \in[a, x]}\left|\int_{t}^{b} g(s) d v(s)+\lambda\right| \bigvee_{a}^{x}(f)+\max _{t \in[x, b]}\left|\int_{a}^{t} g(s) d v(s)-\lambda\right| \bigvee_{x}^{b}(f) \\
& \leq \max \left\{\max _{t \in[a, x]}\left|\int_{t}^{b} g(s) d v(s)+\lambda\right|, \max _{t \in[x, b]}\left|\int_{a}^{t} g(s) d v(s)-\lambda\right|\right\} \bigvee_{a}^{b}(f) .
\end{aligned}
$$

Corollary 4. With the assumptions of Theorem 3 and if the Riemann-Stieltjes integrals below exist, then

$$
\begin{gathered}
\mid f(b) \int_{c}^{b} g(s) d v(s)+f(a) \int_{a}^{d} g(t) d v(t)-f(x) \int_{c}^{d} g(t) d v(t) \\
\quad-\int_{a}^{b} f(t) g(t) d v(t) \mid \\
\leq \max _{t \in[a, x]}\left|\int_{d}^{t} g(s) d v(s)\right| \bigvee_{a}^{x}(f)+\max _{t \in[x, b]}\left|\int_{c}^{t} g(s) d v(s)\right| \bigvee_{x}^{b}(f) \\
\leq \max \left\{\max _{t \in[a, x]}\left|\int_{d}^{t} g(s) d v(s)\right|, \max _{t \in[x, b]}\left|\int_{c}^{t} g(s) d v(s)\right|\right\} \bigvee_{a}^{b}(f) .
\end{gathered}
$$

In particular, for $c=a$ and $d=b$, we have

$$
\begin{aligned}
& \left|[f(b)+f(a)-f(x)] \int_{a}^{b} g(t) d v(t)-\int_{a}^{b} f(t) g(t) d v(t)\right| \\
& \leq \max _{t \in[a, x]}\left|\int_{t}^{b} g(s) d v(s)\right| \bigvee_{a}^{x}(f)+\max _{t \in[x, b]}\left|\int_{a}^{t} g(s) d v(s)\right| \bigvee_{x}^{b}(f) \\
& \leq \max \left\{\max _{t \in[a, x]}\left|\int_{t}^{b} g(s) d v(s)\right|, \max _{t \in[x, b]}\left|\int_{a}^{t} g(s) d v(s)\right|\right\} \bigvee_{a}^{b}(f)
\end{aligned}
$$

for $c=b$ and $d=a$, we have

$$
\begin{aligned}
& \left|f(x) \int_{a}^{b} g(t) d v(t)-\int_{a}^{b} f(t) g(t) d v(t)\right| \\
& \leq \max _{t \in[a, x]}\left|\int_{a}^{t} g(s) d v(s)\right| \bigvee_{a}^{x}(f)+\max _{t \in[x, b]}\left|\int_{t}^{b} g(s) d v(s)\right| \bigvee_{x}^{b}(f) \\
& \leq \max \left\{\max _{t \in[a, x]}\left|\int_{a}^{t} g(s) d v(s)\right|, \max _{t \in[x, b]}\left|\int_{t}^{b} g(s) d v(s)\right|\right\} \bigvee_{a}^{b}(f)
\end{aligned}
$$


and for $c=d=x$

$$
\begin{array}{r}
\left|f(b) \int_{x}^{b} g(s) d v(s)+f(a) \int_{a}^{x} g(t) d v(t)-\int_{a}^{b} f(t) g(t) d v(t)\right| \\
\leq \max _{t \in[a, x]}\left|\int_{t}^{x} g(s) d v(s)\right| \bigvee_{a}^{x}(f)+\max _{t \in[x, b]}\left|\int_{x}^{t} g(s) d v(s)\right| \bigvee_{x}^{b}(f) \\
\leq \max _{t \in[a, b]}\left|\int_{t}^{x} g(s) d v(s)\right| \bigvee_{a}^{b}(f) .
\end{array}
$$

Remark 8. If $m \in(a, b)$ is such that $\bigvee_{a}^{m}(f)=\bigvee_{m}^{b}(f)$, then under the assumptions of Corollary 4, we have

$$
\begin{aligned}
\mid f(b) \int_{c}^{b} g(s) d v(s)+ & f(a) \int_{a}^{d} g(t) d v(t)-f(m) \int_{c}^{d} g(t) d v(t) \\
& -\int_{a}^{b} f(t) g(t) d v(t) \mid \\
\leq & \frac{1}{2}\left[\max _{t \in[a, m]}\left|\int_{d}^{t} g(s) d v(s)\right|+\max _{t \in[m, b]}\left|\int_{c}^{t} g(s) d v(s)\right|\right] \bigvee_{a}^{b}(f),
\end{aligned}
$$

$$
\begin{aligned}
\mid[f(b)+f & (a)-f(m)] \int_{a}^{b} g(t) d v(t)-\int_{a}^{b} f(t) g(t) d v(t) \mid \\
\leq & \frac{1}{2}\left[\max _{t \in[a, m]}\left|\int_{t}^{b} g(s) d v(s)\right|+\max _{t \in[m, b]}\left|\int_{a}^{t} g(s) d v(s)\right|\right] \bigvee_{a}^{b}(f),
\end{aligned}
$$

$$
\begin{aligned}
& \left|f(m) \int_{a}^{b} g(t) d v(t)-\int_{a}^{b} f(t) g(t) d v(t)\right| \\
& \leq \frac{1}{2} \max \left\{\max _{t \in[a, m]}\left|\int_{a}^{t} g(s) d v(s)\right|, \max _{t \in[m, b]}\left|\int_{t}^{b} g(s) d v(s)\right|\right\} \bigvee_{a}^{b}(f)
\end{aligned}
$$

and

$$
\begin{aligned}
\left|f(b) \int_{m}^{b} g(s) d v(s)+f(a) \int_{a}^{m} g(t) d v(t)-\int_{a}^{b} f(t) g(t) d v(t)\right| \\
\leq \frac{1}{2}\left[\max _{t \in[a, m]}\left|\int_{t}^{m} g(s) d v(s)\right|+\max _{t \in[m, b]}\left|\int_{m}^{t} g(s) d v(s)\right|\right] \bigvee_{a}^{b}(f) .
\end{aligned}
$$

Using the equalities (2.9) and (2.10) one can obtain various inequalities as in the recent paper [26]. The details are omitted. 


\section{Inequalities for Lipschitzian Integrands}

We say that the function $f:[a, b] \rightarrow \mathbb{C}$ is Lipschitzian with constant $L>0$ if

$$
|f(t)-f(s)| \leq L|t-s| \text { for all } t, s \in[a, b] .
$$

Theorem 4. Assume that $f:[a, b] \rightarrow \mathbb{C}$ is Lipschitzian with constant $L>0, g$, $v:[a, b] \rightarrow \mathbb{C}, \lambda, \mu \in \mathbb{C}$ and $x, c, d \in[a, b]$ are such that the Riemann-Stieltjes integrals below exist. Then

$$
\begin{aligned}
& \mid\left(\int_{d}^{b} g(s) d v(s)-\mu\right) f(b)+\left(\lambda+\int_{a}^{c} g(t) d v(t)\right) f(a) \\
& +\left(\int_{c}^{d} g(t) d v(t)+\mu-\lambda\right) f(x)-\int_{a}^{b} f(t) g(t) d v(t) \mid \\
& \leq L\left[\max _{t \in[a, x]}\left|\int_{c}^{t} g(s) d v(s)-\lambda\right|(x-a)+\max _{t \in[x, b]}\left|\int_{d}^{t} g(s) d v(s)-\mu\right|(b-x)\right] \\
& \leq L \max \left\{\max _{t \in[a, x]}\left|\int_{c}^{t} g(s) d v(s)-\lambda\right|, \max _{t \in[x, b]}\left|\int_{d}^{t} g(s) d v(s)-\mu\right|\right\}(b-a) .
\end{aligned}
$$

In particular, for $\mu=\lambda$ we have

$$
\begin{gathered}
\mid\left(\int_{d}^{b} g(s) d v(s)-\lambda\right) f(b)+\left(\lambda+\int_{a}^{c} g(t) d v(t)\right) f(a) \\
+f(x) \int_{c}^{d} g(t) d v(t)-\int_{a}^{b} f(t) g(t) d v(t) \mid \\
\leq L\left[\max _{t \in[a, x]}\left|\int_{c}^{t} g(s) d v(s)-\lambda\right|(x-a)+\max _{t \in[x, b]}\left|\int_{d}^{t} g(s) d v(s)-\lambda\right|(b-x)\right] \\
\leq L \max \left\{\max _{t \in[a, x]}\left|\int_{c}^{t} g(s) d v(s)-\lambda\right|, \max _{t \in[x, b]}\left|\int_{d}^{t} g(s) d v(s)-\lambda\right|\right\}(b-a) .
\end{gathered}
$$

Proof. It is well known that, if $p:[a, b] \rightarrow \mathbb{C}$ is Riemann integrable and $v:[a, b] \rightarrow \mathbb{C}$ is Lipschitzian with the constant $L>0$, then

$$
\left|\int_{a}^{b} p(t) d v(t)\right| \leq L \int_{a}^{b}|p(t)| d t .
$$


By using the identity (2.2) and the property (4.4) we get

$$
\begin{aligned}
& \mid\left(\int_{d}^{b} g(s) d v(s)-\mu\right) f(b)+\left(\lambda+\int_{a}^{c} g(t) d v(t)\right) f(a) \\
& \quad+\left(\int_{c}^{d} g(t) d v(t)+\mu-\lambda\right) f(x)-\int_{a}^{b} f(t) g(t) d v(t) \mid \\
& \leq\left|\int_{a}^{x}\left(\int_{c}^{t} g(s) d v(s)-\lambda\right) d f(t)\right|+\left|\int_{x}^{b}\left(\int_{d}^{t} g(s) d v(s)-\mu\right) d f(t)\right| \\
& \quad \leq L \int_{a}^{x}\left|\int_{c}^{t} g(s) d v(s)-\lambda\right| d t+L \int_{x}^{b}\left|\int_{d}^{t} g(s) d v(s)-\mu\right| d t \\
& \leq L \max _{t \in[a, x]}\left|\int_{c}^{t} g(s) d v(s)-\lambda\right|(x-a)+\max _{t \in[x, b]}\left|\int_{d}^{t} g(s) d v(s)-\mu\right|(b-x),
\end{aligned}
$$

which proves the first inequality in (4.2).

The rest is obvious.

Remark 9. For $x=\frac{a+b}{2}$ in (4.2) and (4.3) we get, under the assumptions of Theorem 4, that

$$
\begin{aligned}
& \mid\left(\int_{d}^{b} g(s) d v(s)-\mu\right) f(b)+\left(\lambda+\int_{a}^{c} g(t) d v(t)\right) f(a) \\
& \quad+\left(\int_{c}^{d} g(t) d v(t)+\mu-\lambda\right) f\left(\frac{a+b}{2}\right)-\int_{a}^{b} f(t) g(t) d v(t) \mid \\
& \leq \frac{1}{2} L\left[\max _{t \in\left[a, \frac{a+b}{2}\right]}\left|\int_{c}^{t} g(s) d v(s)-\lambda\right|+\max _{t \in\left[\frac{a+b}{2}, b\right]}\left|\int_{d}^{t} g(s) d v(s)-\mu\right|\right](b-a) .
\end{aligned}
$$

In particular, for $\mu=\lambda$ we have

$$
\begin{aligned}
& \mid\left(\int_{d}^{b} g(s) d v(s)-\lambda\right) f(b)+\left(\lambda+\int_{a}^{c} g(t) d v(t)\right) f(a) \\
& +f\left(\frac{a+b}{2}\right) \int_{c}^{d} g(t) d v(t)-\int_{a}^{b} f(t) g(t) d v(t) \mid \\
& \leq \frac{1}{2} L\left[\max _{t \in\left[a, \frac{a+b}{2}\right]}\left|\int_{c}^{t} g(s) d v(s)-\lambda\right|+\max _{t \in\left[\frac{a+b}{2}, b\right]}\left|\int_{d}^{t} g(s) d v(s)-\lambda\right|\right](b-a) .
\end{aligned}
$$


Corollary 5. With the assumptions of Theorem 4, and if $d=c$, then

$$
\begin{aligned}
& \mid\left(\int_{c}^{b} g(s) d v(s)-\mu\right) f(b)+\left(\lambda+\int_{a}^{c} g(t) d v(t)\right) f(a) \\
& +(\mu-\lambda) f(x)-\int_{a}^{b} f(t) g(t) d v(t) \mid \\
& \leq \max _{t \in[a, x]}\left|\int_{c}^{t} g(s) d v(s)-\lambda\right| \bigvee_{a}^{x}(f)+\max _{t \in[x, b]}\left|\int_{c}^{t} g(s) d v(s)-\mu\right| \bigvee_{x}^{b}(f) \\
& \leq \max \left\{\max _{t \in[a, x]}\left|\int_{c}^{t} g(s) d v(s)-\lambda\right|, \max _{t \in[x, b]}\left|\int_{c}^{t} g(s) d v(s)-\mu\right|\right\} \bigvee_{a}^{b}(f) .
\end{aligned}
$$

In particular, for $\mu=\lambda$, we get

$$
\begin{aligned}
& \mid\left(\int_{c}^{b} g(s) d v(s)-\lambda\right) f(b)+\left(\lambda+\int_{a}^{c} g(t) d v(t)\right) f(a) \\
& -\int_{a}^{b} f(t) g(t) d v(t) \mid \\
& \leq L\left[\max _{t \in[a, x]}\left|\int_{c}^{t} g(s) d v(s)-\lambda\right|(x-a)+\max _{t \in[x, b]}\left|\int_{c}^{t} g(s) d v(s)-\lambda\right|(b-x)\right] \\
& \leq L \max \left\{\max _{t \in[a, x]}\left|\int_{c}^{t} g(s) d v(s)-\lambda\right|, \max _{t \in[x, b]}\left|\int_{c}^{t} g(s) d v(s)-\lambda\right|\right\}(b-a) .
\end{aligned}
$$

Remark 10. If we take $c=x$ in Corollary 5 , then we get

$$
\begin{gathered}
\mid\left(\int_{x}^{b} g(s) d v(s)-\mu\right) f(b)+\left(\lambda+\int_{a}^{x} g(t) d v(t)\right) f(a) \\
+(\mu-\lambda) f(x)-\int_{a}^{b} f(t) g(t) d v(t) \mid \\
\leq L\left[\max _{t \in[a, x]}\left|\int_{x}^{t} g(s) d v(s)-\lambda\right|(x-a)+\max _{t \in[x, b]}\left|\int_{x}^{t} g(s) d v(s)-\mu\right|(b-x)\right] \\
\leq L \max \left\{\max _{t \in[a, x]}\left|\int_{x}^{t} g(s) d v(s)-\lambda\right|, \max _{t \in[x, b]}\left|\int_{x}^{t} g(s) d v(s)-\mu\right|\right\}(b-a) .
\end{gathered}
$$


Preprints (www.preprints.org) | NOT PEER-REVIEWED | Posted: 4 September 2018

doi:10.20944/preprints201809.0060.v1

THREE POINTS INEQUALITIES

17

In particular, for $\mu=\lambda$, we get

$$
\begin{array}{r}
\mid\left(\int_{x}^{b} g(s) d v(s)-\lambda\right) f(b)+\left(\lambda+\int_{a}^{x} g(t) d v(t)\right) f(a) \\
-\int_{a}^{b} f(t) g(t) d v(t) \mid \\
\leq L\left[\max _{t \in[a, x]}\left|\int_{x}^{t} g(s) d v(s)-\lambda\right|(x-a)+\max _{t \in[x, b]}\left|\int_{x}^{t} g(s) d v(s)-\lambda\right|(b-x)\right] \\
\leq L \max _{t \in[a, b]}\left|\int_{x}^{t} g(s) d v(s)-\lambda\right|(b-a) .
\end{array}
$$

Corollary 6. With the assumptions of Theorem 4, and if $c=b$ and $d=a$, then

$$
\begin{aligned}
& \mid\left(\int_{a}^{b} g(s) d v(s)-\mu\right) f(b)+\left(\lambda+\int_{a}^{b} g(t) d v(t)\right) f(a) \\
& +\left(\mu-\lambda-\int_{a}^{b} g(t) d v(t)\right) f(x)-\int_{a}^{b} f(t) g(t) d v(t) \mid \\
& \leq L\left[\max _{t \in[a, x]}\left|\int_{t}^{b} g(s) d v(s)+\lambda\right|(x-a)+\max _{t \in[x, b]}\left|\int_{a}^{t} g(s) d v(s)-\mu\right|(b-x)\right] \\
& \leq L \max \left\{\max _{t \in[a, x]}\left|\int_{t}^{b} g(s) d v(s)+\lambda\right|, \max _{t \in[x, b]}\left|\int_{a}^{t} g(s) d v(s)-\mu\right|\right\}(b-a) .
\end{aligned}
$$

In particular, for $\mu=\lambda$ we have

$$
\begin{gathered}
\mid\left(\int_{a}^{b} g(s) d v(s)-\lambda\right) f(b)+\left(\lambda+\int_{a}^{b} g(t) d v(t)\right) f(a) \\
-f(x) \int_{a}^{b} g(t) d v(t)-\int_{a}^{b} f(t) g(t) d v(t) \mid \\
\leq L\left[\max _{t \in[a, x]}\left|\int_{t}^{b} g(s) d v(s)+\lambda\right|(x-a)+\max _{t \in[x, b]}\left|\int_{a}^{t} g(s) d v(s)-\lambda\right|(b-x)\right] \\
\leq L \max \left\{\max _{t \in[a, x]}\left|\int_{t}^{b} g(s) d v(s)+\lambda\right|, \max _{t \in[x, b]}\left|\int_{a}^{t} g(s) d v(s)-\lambda\right|\right\}(b-a) .
\end{gathered}
$$


Corollary 7. With the assumptions of Theorem 4 and if the Riemann-Stieltjes integrals below exist, then

$$
\begin{gathered}
\mid f(b) \int_{c}^{b} g(s) d v(s)+f(a) \int_{a}^{d} g(t) d v(t)-f(x) \int_{c}^{d} g(t) d v(t) \\
-\int_{a}^{b} f(t) g(t) d v(t) \mid \\
\leq L\left[\max _{t \in[a, x]}\left|\int_{d}^{t} g(s) d v(s)\right|(x-a)+\max _{t \in[x, b]}\left|\int_{c}^{t} g(s) d v(s)\right|(b-x)\right] \\
\leq L \max \left\{\max _{t \in[a, x]}\left|\int_{d}^{t} g(s) d v(s)\right|, \max _{t \in[x, b]}\left|\int_{c}^{t} g(s) d v(s)\right|\right\}(b-a) .
\end{gathered}
$$

In particular, for $c=a$ and $d=b$, we have

$$
\begin{aligned}
& \left|[f(b)+f(a)-f(x)] \int_{a}^{b} g(t) d v(t)-\int_{a}^{b} f(t) g(t) d v(t)\right| \\
& \leq \max _{t \in[a, x]}\left|\int_{t}^{b} g(s) d v(s)\right| \bigvee_{a}^{x}(f)+\max _{t \in[x, b]}\left|\int_{a}^{t} g(s) d v(s)\right| \bigvee_{x}^{b}(f) \\
& \leq \max \left\{\max _{t \in[a, x]}\left|\int_{t}^{b} g(s) d v(s)\right|, \max _{t \in[x, b]}\left|\int_{a}^{t} g(s) d v(s)\right|\right\} \bigvee_{a}^{b}(f)
\end{aligned}
$$

for $c=b$ and $d=a$, we have

$$
\begin{aligned}
& \left|f(x) \int_{a}^{b} g(t) d v(t)-\int_{a}^{b} f(t) g(t) d v(t)\right| \\
& \leq L\left[\max _{t \in[a, x]}\left|\int_{a}^{t} g(s) d v(s)\right|(x-a)+\max _{t \in[x, b]}\left|\int_{t}^{b} g(s) d v(s)\right|(b-x)\right] \\
& \leq L \max \left\{\max _{t \in[a, x]}\left|\int_{a}^{t} g(s) d v(s)\right|, \max _{t \in[x, b]}\left|\int_{t}^{b} g(s) d v(s)\right|\right\}(b-a)
\end{aligned}
$$

and for $c=d=x$ we get

$$
\begin{array}{r}
\left|f(b) \int_{x}^{b} g(s) d v(s)+f(a) \int_{a}^{x} g(t) d v(t)-\int_{a}^{b} f(t) g(t) d v(t)\right| \\
\leq L\left[\max _{t \in[a, x]}\left|\int_{t}^{x} g(s) d v(s)\right|(x-a)+\max _{t \in[x, b]}\left|\int_{x}^{t} g(s) d v(s)\right|(b-x)\right] \\
\leq L \max _{t \in[a, b]}\left|\int_{t}^{x} g(s) d v(s)\right|(b-a) .
\end{array}
$$


Remark 11. If we take $x=\frac{a+b}{2}$, then under the assumptions of Corollary 7, we have

$$
\begin{aligned}
\mid f(b) \int_{c}^{b} g(s) d v(s)+ & f(a) \int_{a}^{d} g(t) d v(t)-f\left(\frac{a+b}{2}\right) \int_{c}^{d} g(t) d v(t) \\
& -\int_{a}^{b} f(t) g(t) d v(t) \mid \\
\leq & \frac{1}{2} L\left[\max _{t \in\left[a, \frac{a+b}{2}\right]}\left|\int_{d}^{t} g(s) d v(s)\right|+\max _{t \in\left[\frac{a+b}{2}, b\right]}\left|\int_{c}^{t} g(s) d v(s)\right|\right](b-a),
\end{aligned}
$$

$$
\begin{aligned}
& \left|\left[f(b)+f(a)-f\left(\frac{a+b}{2}\right)\right] \int_{a}^{b} g(t) d v(t)-\int_{a}^{b} f(t) g(t) d v(t)\right| \\
& \quad \leq \frac{1}{2} L\left[\max _{t \in\left[a, \frac{a+b}{2}\right]}\left|\int_{t}^{b} g(s) d v(s)\right|+\max _{t \in\left[\frac{a+b}{2}, b\right]}\left|\int_{a}^{t} g(s) d v(s)\right|\right](b-a),
\end{aligned}
$$

$$
\begin{aligned}
& \left|f\left(\frac{a+b}{2}\right) \int_{a}^{b} g(t) d v(t)-\int_{a}^{b} f(t) g(t) d v(t)\right| \\
& \leq \frac{1}{2} L \max \left\{\max _{t \in\left[a, \frac{a+b}{2}\right]}\left|\int_{a}^{t} g(s) d v(s)\right|, \max _{t \in\left[\frac{a+b}{2}, b\right]}\left|\int_{t}^{b} g(s) d v(s)\right|\right\}(b-a)
\end{aligned}
$$

and

$$
\begin{aligned}
& \left|f(b) \int_{\frac{a+b}{2}}^{b} g(s) d v(s)+f(a) \int_{a}^{\frac{a+b}{2}} g(t) d v(t)-\int_{a}^{b} f(t) g(t) d v(t)\right| \\
& \leq \frac{1}{2} L\left[\max _{t \in\left[a, \frac{a+b}{2}\right]}\left|\int_{t}^{\frac{a+b}{2}} g(s) d v(s)\right|+\max _{t \in\left[\frac{a+b}{2}, b\right]}\left|\int_{\frac{a+b}{2}}^{t} g(s) d v(s)\right|\right](b-a) .
\end{aligned}
$$

\section{Some Simpler Error Bounds}

If $g:[a, b] \rightarrow \mathbb{C}$ is continuous and $v:[a, b] \rightarrow \mathbb{C}$ is of bounded variation, then the Riemann-Stieltjes integrals $\int_{a}^{t} g(s) d v(s)$ and $\int_{t}^{b} g(s) d v(s)$ exist for $t \in[a, b]$ and

$$
\left|\int_{a}^{t} g(s) d v(s)\right| \leq \int_{a}^{t}|g(s)| d\left(\bigvee_{a}^{s}(v)\right) \leq \max _{s \in[a, t]}|g(s)| \bigvee_{a}^{t}(v)
$$

and

$$
\left|\int_{t}^{b} g(s) d v(s)\right| \leq \int_{t}^{b}|g(s)| d\left(\bigvee_{t}^{s}(v)\right) \leq \max _{s \in[t, b]}|g(s)| \bigvee_{t}^{b}(v),
$$

which implies that

$$
\max _{t \in[a, x]}\left|\int_{a}^{t} g(s) d v(s)\right| \leq \max _{s \in[a, x]}|g(s)| \bigvee_{a}^{x}(v)
$$

and

$$
\max _{t \in[x, b]}\left|\int_{t}^{b} g(s) d v(s)\right| \leq \max _{s \in[x, b]}|g(s)| \bigvee_{x}^{b}(v)
$$


for $x \in(a, b)$.

Therefore, by (3.14) we get for $x \in(a, b)$ that

$$
\begin{aligned}
\mid f(x) & \int_{a}^{b} g(t) d v(t)-\int_{a}^{b} f(t) g(t) d v(t) \mid \\
\leq & \max _{t \in[a, x]}\left|\int_{a}^{t} g(s) d v(s)\right| \bigvee_{a}^{x}(f)+\max _{t \in[x, b]}\left|\int_{t}^{b} g(s) d v(s)\right| \bigvee_{x}^{b}(f) \\
& \leq \max _{s \in[a, x]}|g(s)| \bigvee_{a}^{x}(v) \bigvee_{a}^{x}(f)+\max _{s \in[x, b]}|g(s)| \bigvee_{x}^{b}(v) \bigvee_{x}^{b}(f) \\
\leq & \left\{\begin{array}{l}
\max _{x}\left\{\max _{s \in[a, x]}|g(s)| \bigvee_{a}^{x}(v), \max _{s \in[x, b]}|g(s)| \bigvee_{x}^{b}(v)\right\} \bigvee_{a}^{b}(f), \\
\max \left\{\max _{s \in[a, x]}|g(s)| \bigvee_{a}^{x}(f), \max _{s \in[x, b]}|g(s)| \bigvee_{x}^{b}(f)\right\} \bigvee_{a}^{b}(v)
\end{array}\right.
\end{aligned}
$$

provided $f, v$ are of bounded variation and $g$ is continuous and such that the integral $\int_{a}^{b} f(t) g(t) d v(t)$ exists.

If $m \in(a, b)$ is such that $\bigvee_{a}^{m}(f)=\bigvee_{m}^{b}(f)$, then from the first inequality in (5.1) we get

$$
\begin{aligned}
\left|f(m) \int_{a}^{b} g(t) d v(t)-\int_{a}^{b} f(t) g(t) d v(t)\right| \\
\leq \frac{1}{2}\left[\max _{t \in[a, m]}\left|\int_{a}^{t} g(s) d v(s)\right|+\max _{t \in[m, b]}\left|\int_{t}^{b} g(s) d v(s)\right|\right] \bigvee_{a}^{b}(f) .
\end{aligned}
$$

If $p \in(a, b)$ is such that $\bigvee_{a}^{p}(v)=\bigvee_{p}^{b}(v)$, then from the inequality (5.1) we get

$$
\begin{aligned}
& \mid f(p) \int_{a}^{b} g(t) d v(t)-\int_{a}^{b} f(t) g(t) d v(t) \mid \\
& \leq \max _{t \in[a, p]}\left|\int_{a}^{t} g(s) d v(s)\right| \bigvee_{a}^{p}(f)+\max _{t \in[p, b]}\left|\int_{t}^{b} g(s) d v(s)\right| \bigvee_{p}^{b}(f) \\
& \leq \frac{1}{2}\left[\max _{s \in[a, p]}|g(s)| \bigvee_{a}^{p}(f)+\max _{s \in[p, b]}|g(s)| \bigvee_{p}^{b}(f)\right] \bigvee_{a}^{b}(v)
\end{aligned}
$$


By (4.15) we also get for $x \in(a, b)$ that

$$
\begin{aligned}
& \left|f(x) \int_{a}^{b} g(t) d v(t)-\int_{a}^{b} f(t) g(t) d v(t)\right| \\
& \leq L\left[\max _{t \in[a, x]}\left|\int_{a}^{t} g(s) d v(s)\right|(x-a)+\max _{t \in[x, b]}\left|\int_{t}^{b} g(s) d v(s)\right|(b-x)\right] \\
& \leq L\left[\max _{s \in[a, x]}|g(s)| \bigvee_{a}^{x}(v)(x-a)+\max _{s \in[x, b]}|g(s)| \bigvee_{x}^{b}(v)(b-x)\right] \\
& \leq L\left\{\begin{array}{l}
\max _{a}\left\{\max _{s \in[a, x]}|g(s)| \bigvee_{a}^{x}(v), \max _{s \in[x, b]}|g(s)| \bigvee_{x}^{b}(v)\right\}(b-a), \\
\max \left\{\max _{s \in[a, x]}|g(s)|(x-a), \max _{s \in[x, b]}|g(s)|(b-x)\right\} \bigvee_{a}^{b}(v)
\end{array}\right.
\end{aligned}
$$

provided $v$ is of bounded variation, $f$ is Lipschitzian with the constant $L>0$ and $g$ is continuous on $[a, b]$.

In particular, for $x=\frac{a+b}{2}$ we get from the first inequality in (5.4) that

$$
\begin{aligned}
& \left|f\left(\frac{a+b}{2}\right) \int_{a}^{b} g(t) d v(t)-\int_{a}^{b} f(t) g(t) d v(t)\right| \\
& \quad \leq \frac{1}{2} L\left[\max _{t \in\left[a, \frac{a+b}{2}\right]}\left|\int_{a}^{t} g(s) d v(s)\right|+\max _{t \in\left[\frac{a+b}{2}, b\right]}\left|\int_{t}^{b} g(s) d v(s)\right|\right](b-a) .
\end{aligned}
$$

$$
\begin{aligned}
& \left|f(p) \int_{a}^{b} g(t) d v(t)-\int_{a}^{b} f(t) g(t) d v(t)\right| \\
& \leq L\left[\max _{t \in[a, p]}\left|\int_{a}^{t} g(s) d v(s)\right|(p-a)+\max _{t \in[p, b]}\left|\int_{t}^{b} g(s) d v(s)\right|(b-p)\right] \\
& \leq \frac{1}{2} L\left[\max _{s \in[a, p]}|g(s)|(p-a)+\max _{s \in[p, b]}|g(s)|(b-p)\right] \bigvee_{a}^{b}(v) .
\end{aligned}
$$

If $p \in(a, b)$ is such that $\bigvee_{a}^{p}(v)=\bigvee_{p}^{b}(v)$, then from the inequality (5.4) we get 
Similarly, by (3.15) we have for $x \in(a, b)$ that

$$
\begin{aligned}
\mid f(b) & \int_{x}^{b} g(s) d v(s)+f(a) \int_{a}^{x} g(t) d v(t)-\int_{a}^{b} f(t) g(t) d v(t) \mid \\
\leq & \max _{t \in[a, x]}\left|\int_{t}^{x} g(s) d v(s)\right| \bigvee_{a}^{x}(f)+\max _{t \in[x, b]}\left|\int_{x}^{t} g(s) d v(s)\right| \bigvee_{x}^{b}(f) \\
& \leq \max _{s \in[a, x]}|g(s)| \bigvee_{a}^{x}(v) \bigvee_{a}^{x}(f)+\max _{s \in[x, b]}|g(s)| \bigvee_{x}^{b}(v) \bigvee_{x}^{b}(f) \\
& \leq\left\{\begin{array}{l}
\max _{x}\left\{\max _{s \in[a, x]}|g(s)| \bigvee_{a}^{x}(v), \max _{s \in[x, b]}|g(s)| \bigvee_{x}^{b}(v)\right\} \bigvee_{a}^{b}(f) \\
\max _{x}^{x}\left\{\max _{s \in[a, x]}|g(s)| \bigvee_{a}^{b}(f), \max _{s \in[x, b]}|g(s)| \bigvee_{x}^{b}(f)\right\} \bigvee_{a}^{b}(v)
\end{array}\right.
\end{aligned}
$$

provided $f, v$ are of bounded variation and $g$ is continuous and such that the integral $\int_{a}^{b} f(t) g(t) d v(t)$ exists.

If $m \in(a, b)$ is such that $\bigvee_{a}^{m}(f)=\bigvee_{m}^{b}(f)$, then from the first inequality in (5.7) we get

$$
\begin{aligned}
\left|f(b) \int_{m}^{b} g(s) d v(s)+f(a) \int_{a}^{m} g(t) d v(t)-\int_{a}^{b} f(t) g(t) d v(t)\right| \\
\leq \frac{1}{2}\left[\max _{t \in[a, m]}\left|\int_{t}^{m} g(s) d v(s)\right|+\max _{t \in[m, b]}\left|\int_{m}^{t} g(s) d v(s)\right|\right] \bigvee_{a}^{b}(f) .
\end{aligned}
$$

If $p \in(a, b)$ is such that $\bigvee_{a}^{p}(v)=\bigvee_{p}^{b}(v)$, then from the inequality (5.1) we get

$$
\begin{aligned}
& \mid f(b) \int_{p}^{b} g(s) d v(s)+f(a) \int_{a}^{p} g(t) d v(t)-\int_{a}^{b} f(t) g(t) d v(t) \mid \\
& \leq \max _{t \in[a, p]}\left|\int_{t}^{p} g(s) d v(s)\right| \bigvee_{a}^{p}(f)+\max _{t \in[p, b]}\left|\int_{p}^{t} g(s) d v(s)\right| \bigvee_{p}^{b}(f) \\
& \leq \frac{1}{2}\left[\max _{s \in[a, p]}|g(s)| \bigvee_{a}^{p}(f)+\max _{s \in[p, b]}|g(s)| \bigvee_{p}^{b}(f)\right] \bigvee_{a}^{b}(v) .
\end{aligned}
$$


From (4.16) we have

$$
\begin{aligned}
& \left|f(b) \int_{x}^{b} g(s) d v(s)+f(a) \int_{a}^{x} g(t) d v(t)-\int_{a}^{b} f(t) g(t) d v(t)\right| \\
& \leq L\left[\max _{t \in[a, x]}\left|\int_{t}^{x} g(s) d v(s)\right|(x-a)+\max _{t \in[x, b]}\left|\int_{x}^{t} g(s) d v(s)\right|(b-x)\right] \\
& \leq L\left[\max _{s \in[a, x]}|g(s)| \bigvee_{a}^{x}(v)(x-a)+\max _{s \in[x, b]}|g(s)| \bigvee_{x}^{b}(v)(b-x)\right] \\
& \leq L\left\{\max _{x}^{x}\left\{\max _{s \in[a, x]}|g(s)| \bigvee_{a}(v), \max _{s \in[x, b]}|g(s)| \bigvee_{x}^{b}(v)\right\}(b-a),\right. \\
& \max \left\{\max _{s \in[a, x]}|g(s)|(x-a), \max _{s \in[x, b]}|g(s)|(b-x)\right\} \bigvee_{a}^{b}(v)
\end{aligned}
$$

provided that $v$ is of bounded variation, $f$ is Lipschitzian with the constant $L>0$ and $g$ is continuous on $[a, b]$.

In particular, for $x=\frac{a+b}{2}$ we get from the first inequality in (5.10) that

$$
\begin{aligned}
& \left|f(b) \int_{\frac{a+b}{2}}^{b} g(s) d v(s)+f(a) \int_{a}^{\frac{a+b}{2}} g(t) d v(t)-\int_{a}^{b} f(t) g(t) d v(t)\right| \\
& \leq \frac{1}{2} L\left[\max _{t \in\left[a, \frac{a+b}{2}\right]}\left|\int_{t}^{\frac{a+b}{2}} g(s) d v(s)\right|+\max _{t \in\left[\frac{a+b}{2}, b\right]}\left|\int_{\frac{a+b}{2}}^{t} g(s) d v(s)\right|\right](b-a) .
\end{aligned}
$$

If $p \in(a, b)$ is such that $\bigvee_{a}^{p}(v)=\bigvee_{p}^{b}(v)$, then from the inequality (5.10) we get

$$
\begin{aligned}
& \left|f(b) \int_{p}^{b} g(s) d v(s)+f(a) \int_{a}^{p} g(t) d v(t)-\int_{a}^{b} f(t) g(t) d v(t)\right| \\
& \leq L\left[\max _{t \in[a, p]}\left|\int_{t}^{p} g(s) d v(s)\right|(p-a)+\max _{t \in[p, b]}\left|\int_{p}^{t} g(s) d v(s)\right|(b-p)\right] \\
& \leq \frac{1}{2} L\left[\max _{s \in[a, x]}|g(s)|(p-a)+\max _{s \in[x, b]}|g(s)|(b-p)\right] \bigvee_{a}^{b}(v) .
\end{aligned}
$$

Using the equalities (2.9) and (2.10) one can obtain various inequalities as in the recent paper [27]. The details are omitted.

\section{REFERENCES}

[1] T. M. Apostol, Mathematical Analysis, Addison-Wesley Publishing Company, Second Edition, 1981.

[2] N. S. Barnett, W. S. Cheung, S. S. Dragomir and A. Sofo, Ostrowski and trapezoid type inequalities for the Stieltjes integral with Lipschitzian integrands or integrators, Comput. Math. Appl. 57 (2009), no. 2, 195-201. Preprint RGMIA Res. Rep. Coll. 9 (2006), No. 4, Article 9. 
[3] P. Cerone, W. S. Cheung and S. S. Dragomir, On Ostrowski type inequalities for Stieltjes integrals with absolutely continuous integrands and integrators of bounded variation, Comput. Math. Appl. 54 (2007), no. 2, 183-191. Preprint RGMIA Res. Rep. Coll. 9 (2006), No. 2, Article 14. [ONLINE: http://rgmia.vu.edu.au/v9n2.html].

[4] P. Cerone and S. S. Dragomir, Trapezoid type rules from an inequalities point of view, in Handbook of Analytic Computational Methods in Applied Mathematics, Ed. G. Anastassiou, CRC Press, New York, pp. 65-134.

[5] P. Cerone and S. S. Dragomir, A refinement of the Grüss inequality and applications, Tamkang J. Math. 38 (2007), no. 1, 37-49. Preprint RGMIA Res. Rep. Coll., 5 (2) (2002), Article 14.

[6] P. Cerone, S. S. Dragomir and C. E. M. Pearce, A generalised trapezoid inequality for functions of bounded variation, Turkish J. Math., 24 (2) (2000), 147-163.

[7] X. L. Cheng and J. Sun, A note on the perturbed trapezoid inequality, J. Ineq. Pure and Appl. Math., 3(2) Art. 29, (2002).

[8] W. S. Cheung and S. S. Dragomir, Two Ostrowski type inequalities for the Stieltjes integral of monotonic functions, Bull. Austral. Math. Soc. 75 (2007), no. 2, 299-311., Preprint RGMIA Res. Rep. Coll. 9 (2006), No. 3, Article 8.

[9] W. S. Cheung and S. S. Dragomir, A survey on Ostrowski type inequalities for RiemannStieltjes integral. Handbook of functional equations, 75-104, Springer Optim. Appl., 95,Springer, New York, 2014.

[10] S. S. Dragomir, The Ostrowski integral inequality for mappings of bounded variation. Bull. Austral. Math. Soc. 60 (1999), No. 3, 495-508.

[11] S. S. Dragomir, Ostrowski's inequality for monotonous mappings and applications, J. KSIAM, 3 (1) (1999), 127-135.

[12] S. S. Dragomir, The Ostrowski's integral inequality for Lipschitzian mappings and applications, Computers and Math. with Applic., 38 (1999), 33-37.

[13] S. S. Dragomir, A generalization of Grüss's inequality in inner product spaces and applications. J. Math. Anal. Appl. 237 (1999), no. 1, 74-82.

[14] S. S. Dragomir, New Grüss' type inequalities for functions of bounded variation and applications, Applied Mathematics Letters 25 (2012) 1475-1479.

[15] S. S. Dragomir, On the Ostrowski's inequality for Riemann-Stieltjes integral, Korean J. Appl. Math., 7 (2000), 477-485.

[16] S. S. Dragomir, On the Ostrowski's inequality for Riemann-Stieltjes integral $\int_{a}^{b} f(t) d u(t)$ where $f$ is of Hölder type and $u$ is of bounded variation and applications, J. KSIAM, 5 (1) (2001), 35-45.

[17] S. S. Dragomir, On the Ostrowski's integral inequality for mappings with bounded variation and applications, Math. Ineq. Appl. 4 (2001), No. 1, 59-66. Preprint: RGMIA Res. Rep. Coll. 2 (1999), Art. 7.

[18] S. S. Dragomir, The median principle for inequalities and applications. Functional equations, inequalities and applications, 21-37, Kluwer Acad. Publ., Dordrecht, 2003.

[19] S. S. Dragomir, A companion of the Grüss inequality and applications. Appl. Math. Lett. 17 (2004), no. 4, 429-435.

[20] S. S. Dragomir, Inequalities of Grüss type for the Stieltjes integral, Kragujevac J. Math., 26 (2004), 89-122.

[21] S. S. Dragomir, A generalisation of Cerone's identity and applications, Tamsui Oxf. J. Math. Sci. 23 (2007), no. 1, 79-90. Preprint RGMIA Res. Rep. Coll. 8(2005), No. 2. Artcile 19.

[22] S. S. Dragomir, Inequalities for Stieltjes integrals with convex integrators and applications, Appl. Math. Lett., 20 (2007), 123-130.

[23] S. S. Dragomir, The perturbed median principle for integral inequalities with applications. Nonlinear analysis and variational problems, 53-63, Springer Optim. Appl., 35, Springer, New York, 2010.

[24] S. S. Dragomir, Some inequalities for continuous functions of selfadjoint operators in Hilbert spaces, Acta Math Vietnam (2014) 39:287-303, DOI 10.1007/s40306-014-0061-4. Preprint RGMIA Res. Rep. Coll. 15 (2012), Art. 16.

[25] S. S. Dragomir, Ostrowski type inequalities for Lebesgue integral: a survey of recent results. Aust. J. Math. Anal. Appl. 14 (2017), no. 1, Art. 1, 283 pp.

[26] S. S. Dragomir, Three points inequalities for Riemann-Stieltjes integral with integrands and integrators of bounded variation, Preprint RGMIA Res. Rep. Coll. 21 (2018), Art. 103, 24 pp. [Online http://rgmia.org/papers/v21/v21a103.pdf] . 
[27] S. S. Dragomir, New three points inequalities for Riemann-Stieltjes integral of Lipschitzian integrands and integrators of bounded variation, Preprint RGMIA Res. Rep. Coll. 21 (2018), Art. 104, 21 pp. [Online http://rgmia.org/papers/v21/v21a104.pdf] .

[28] S. S. Dragomir, C. Buşe, M. V. Boldea and L. Braescu, A generalisation of the trapezoidal rule for the Riemann-Stieltjes integral and applications, Nonlinear Anal. Forum, (Korea) 6 (2) (2001), 337-351.

[29] S. S. Dragomir and I. Fedotov, An inequality of Grüss type for the Riemann-Stieltjes integral and applications for special means, Tamkang J. Math., 29 (4) (1998), 287-292.

[30] S. S. Dragomir and I. Fedotov, A Grüss type inequality for mappings of bounded variation and applications for numerical analysis, Nonlinear Funct. Anal. Appl., 6 (3) (2001), 425-433.

[31] G. Helmberg, Introduction to Spectral Theory in Hilbert Space, John Wiley \& Sons, Inc. -New York, 1969.

[32] Z. Liu, Refinement of an inequality of Grüss type for Riemann-Stieltjes integral, Soochow J. Math., 30 (4) (2004), 483-489.

[33] A. Ostrowski, Uber die Absolutabweichung einer differentienbaren Funktionen von ihren Integralmittelwert, Comment. Math. Hel, 10 (1938), 226-227.

${ }^{1}$ Mathematics, College of Engineering \& Science, Victoria University, PO Box 14428, Melbourne City, MC 8001, Australia.

E-mail address: sever.dragomir@vu.edu.au

$U R L:$ http://rgmia.org/dragomir

${ }^{2}$ DSt-nRF Centre of Excellence, in the Mathematical and Statistical Sciences, School of Computer Science \& Applied Mathematics, University of the Witwatersrand, Private Bag 3, Johannesburg 2050, South Africa 